\title{
INTEGRACIÓN SOCIOCULTURAL Y DERECHOS DE LAS NIÑAS Y LOS NIÑOS MIGRANTES EN EL CONTEXTO LOCAL. EL CASO DE RECOLETA (REGIÓN METROPOLITANA, CHILE)
}

\author{
SOCIOCULTURAL INTEGRATION AND RIGHTS OF IMMIGRANT \\ CHILDREN IN THE LOCAL CONTEXT. THE CASE OF THE RECOLETA \\ DISTRICT (METROPOLITAN REGION, CHILE)
}

\author{
Iskra Pavez-Soto ${ }^{1,2}$
}

\begin{abstract}
La integración sociocultural que viven las niñas y los niños migrantes es cada vez más un asunto de interés local, puesto que es en la familia, la escuela o el barrio (el territorio) donde se ejercen cotidianamente los derechos y se experimenta el proceso de integración. A partir de este enfoque, este artículo analiza los procesos de integración sociocultural que viven niñas y niños migrantes en Recoleta (Región Metropolitana, Chile). El diseño metodológico de la investigación ha sido cualitativo, a través de entrevistas a niñas y niños migrantes de 6 a 14 años de edad, de Perú y nacidos en Chile de madres y padres extranjeros. Se concluye que en el ámbito local de Recoleta se ejercen algunos derechos sociales (como salud y educación), pero las situaciones de pobreza, exclusión y discriminación afectan gravemente los procesos de integración social. Aunque el derecho a la opinión no siempre es considerado en los asuntos que les afectan -como su propia migración-, cabe destacar su elevada participación en asociaciones locales que fortalecen el sentido de pertenencia y una reflexión crítica con las formas culturales de "ser niña o niño, aquí y ahora".
\end{abstract}

Palabras claves: niñas y niños, migración, integración sociocultural, derechos, Chile.

Sociocultural integration of migrant children is increasingly becoming an issue of local interest, as it is in the family, school and neighbourhood where rights are exercised and integration is experienced. Within this perspective, this article analyzes the sociocultural integration of immigrant children in the Recoleta district in Santiago (Chile). Data for this qualitative study have been collected through interviews with immigrant children ages 6 tol 4 both from Peru and born in Chile to immigrant parents. The study concludes that social rights (like health and education) are exercised in Recoleta. However, social integration is greatly affected by poverty, exclusion and discrimination. Even though their voices are not always heard in matters directly affecting them -like their own migration-, these children actively participate in local associations that strengthen a sense of belonging and promote a critical reflection on the cultural forms of "being a girl and a boy, here and now".

Key words: Children, immigrant, rights, and sociocultural integration, Chile.

Diversos estudios (Márquez 2013; Margarit y Bijit 2014; Stefoni 2015) han demostrado que el territorio es un factor clave en los procesos de integración que viven las personas y comunidades migrantes en Chile. Es que el territorio local es el espacio que acoge a las niñas y los niños migrantes; es significante y significado de la experiencia de habitar, un lugar entre el aquí y el allí, donde se echan raíces y se va haciendo historia (Márquez 2013:322). El objetivo de este artículo es analizar los procesos de integración sociocultural que viven las niñas y los niños migrantes a través del ejercicio de sus derechos en un contexto local específico, Recoleta (Región Metropolitana, Chile). Los resultados presentados forman parte de un estudio FONDECYT.

Según Márquez (2013:324), el territorio conocido actualmente como Recoleta e históricamente como La Chimba, el cual se emplaza al otro lado del Río Mapocho (hacia el norte) ha sido durante siglos un espacio de cobijo de migrantes y, por tanto, un símbolo de la diversidad cultural de la ciudad. Desde los años noventa han comenzado a instalarse familias y comunidades migrantes allí. Según los

\footnotetext{
1 Universidad Bernardo O'Higgins, Centro de Estudios Políticos, Culturales y Sociales de América Latina (EPOCAL), Fábrica No 1990, Santiago, Chile. iskra.pavez@ubo.cl

2 Corporación Opción, Centro de Estudios de la Niñez (CEN), Carlos Justiniano No 1123, Providencia, Santiago, Chile.
} 
últimos registros, residen 44.680 personas extranjeras en Recoleta, principalmente en torno a los barrios comerciales que históricamente han acogido a migrantes, como Patronato, Dorsal y el sector de la Vega Central (Rojas y Silva 2016).

Según un estudio realizado a nivel nacional (Silva et al. 2011), Recoleta se destaca por sus políticas de infancia, ya que cuenta con una Oficina de Protección de Derechos (en adelante OPD), instancia cofinanciada entre el Servicio Nacional de Menores-SENAME y municipio, una Oficina de la Niñez y un Programa de Migrantes (fue uno de los primeros municipios en instalarlo), lo que demuestra voluntad política e interés en garantizar los derechos de las niñas y los niños migrantes en la comuna ${ }^{1}$. Por otro lado, de acuerdo a datos municipales (OPD Recoleta 2013:18), un 4,8\% de niñas y niños que habitan en la comuna son migrantes y provienen principalmente de Perú, Ecuador, China, Argentina y otras nacionalidades, también se registró que las niñas y los niños migrantes tienen una mejor percepción (en comparación con los autóctonos) sobre el respeto de sus derechos. Sin embargo, se sienten aislados y poco acogidos por la comunidad escolar, concretamente dentro de sus grupos de pares, debido a la discriminación que sufren por su condición migrante (OPD Recoleta 2013:21-31). El artículo está estructurado en tres apartados: una primera parte teórica, luego, el análisis y, finalmente, las conclusiones.

\section{Integración Sociocultural: Ajustes Conceptuales}

El concepto de integración es complejo, ya que ineludiblemente refiere a los modelos sociales y culturales dominantes en un determinado territorio. En el ámbito específico de las migraciones, surge la retórica pregunta: ¿a qué deben integrarse las personas migrantes? (Malgesini y Giménez 2000:248 y ss). En Estados Unidos y Europa el concepto de integración de Gordon (1964) -entendido como asimilación de las formas culturales dominantes (anglo-conformity)- tuvo una gran influencia en los estudios migratorios, pero también fue altamente criticado, porque no todos los grupos migrantes viven una asimilación; de hecho, algunos experimentan situaciones permanentes de exclusión social y cultural, por ese motivo autores como Portes et al. (2006) en vez de hablar de integración, prefieren usar el concepto de "asimilación segmentada" dependiendo del estrato al cual se inserten.

El concepto de integración considera tanto las diferencias culturales de las personas y los grupos migrantes, así como las condiciones sociales, económicas y políticas en las cuales viven (Solé et al. 2011). La dimensión social y cultural de la integración quedan muy bien articuladas en el concepto bidimensional de justicia de Fraser (2008:84), quien sostiene que la redistribución igualitaria (el acceso a derechos sociales y económicos) debe ir acompañada necesariamente de políticas de reconocimiento e identidad (derechos políticos y culturales); de modo que, por separado ninguno de los dos ámbitos sería suficiente.

Tradicionalmente se ha definido la cultura como las formas de vida de los grupos y las comunidades, que se presumen homogéneas (García Castaño et al. 1993). No obstante, según Bhabha (2002:21), esa concepción homegeneizante de la cultura o de las comunidades étnicas, está en un profundo proceso de redefinición. Es por esta razón que en este artículo preferimos optar por una compresión de la cultura como una producción desigual e incompleta de sentido y valor, a menudo compuesta de demandas y prácticas inconmensurables. La cultura nacional logra crear una textualidad simbólica y ofrece una identidad, pero esa continuidad histórica y de preservación de las tradiciones también puede ser problemática (Bhabha 2013:110).

\section{La integración sociocultural de las niñas y los niños migrantes}

Etimológicamente la palabra infancia proviene del latín infantǐa, cuyo significado primario alude a la incapacidad de hablar o sin voz (Pavez-Soto 2012a); mientras que la palabra niño o niña presenta una polisemia muy amplia, aunque en general apunta a la condición de las personas con pocos años de edad o quienes se encuentran en posición de subordinación social (servidumbre) (Wasserman $(2001: 61)^{2}$. Desde los estudios socioculturales, Gaitán (2006) define que la infancia sería una condición social delimitada por una construcción cultural e histórica diferenciada y caracterizada por relaciones de poder basadas en la edad; mientras, que las niñas y los niños serían el grupo de personas o sujetos sociales que se desenvuelven en dicho espacio social. Es preciso reconocer que en Chile, particularmente en las leyes y políticas públicas, se 
utilizan como sinónimos los términos infancia, niños (en masculino y plural) y, en ocasiones, menores. De hecho, aún está vigente la llamada Ley de Menores promulgada en 1967, si bien se está legislando un nuevo marco regulador. Para efectos del presente artículo se entenderá que el concepto de infancia apunta hacia el fenómeno social y culturalmente construido y reconstruido por las políticas públicas, mientras que las niñas y los niños son los sujetos sociales que experimentan dicho fenómeno. En otras palabras, se utiliza el concepto de "infancia" cuando se está apuntando al fenómeno social y cultural que es construido de diversas maneras, históricamente a través del tiempo y reconstruido a través de las políticas públicas; mientras que se usa el término "niñas y niños" cuando se está hablando de los sujetos concretos que experimentan dicho fenómeno; no se usan como sinónimos.

Desde la Antropología se entiende que la infancia podría ser vista como un fenómeno construido socioculturalmente, porque representa categorías sociales a la que los grupos, a lo largo del tiempo, le asignan (a la infancia) unos significados u otros. Existen distintas representaciones de lo que es ser niña o niño en las diversas culturas, contextos o campos discursivos concretos; la infancia no es una instancia abstracta y universal, sino que participa de los cambios y las mismas condiciones de existencia de la cultura (Moscoso 2008:5). Complementario con lo anterior, desde una mirada sociológica (Qvortrup 1994), la infancia como un fenómeno, representa un campo en disputa, porque simboliza el espacio y el momento en el cual la comunidad se reproduce a sí misma; las niñas y los niños tienen la misión de darle continuidad a una determinada sociedad y cultura, por eso, generalmente se asocian al futuro (James y James 2012:3).

Comprender el fenómeno de la infancia desde una mirada sociocultural, implica reconocer que las niñas y los niños también participan de las relaciones de género. Entendiendo el género como el conjunto de características sociales, culturales, políticas, psicológicas, jurídicas y económicas asignadas al sexo diferencialmente (Lamas 1999). Los roles de género vigentes en cada contexto cultural se aprenden, justamente, durante la infancia mediante el proceso de socialización, no se derivan "naturalmente" de su sexo y se van reconstruyendo durante todo el ciclo vital.

A partir de esta mirada antropológica y sociológica, resulta evidente que la integración de las niñas y los niños migrantes no solo implica el ejercicio de sus derechos a la educación o la salud, sino, también, la integración en las formas socioculturales de "ser niña o niño, aquí y ahora" (Feixa 2006). Para ser aceptados en sus grupos de pares, eventualmente deberán "imitar" los estilos culturales y el lenguaje de las niñas y los niños autóctonos, aunque esta adaptación implique contradicciones y conflictos generacionales con sus familias (Alegre 2007).

La escuela cumple un rol fundamental en este proceso de integración que viven las niñas y los niños migrantes, porque ahí aprenden el idioma, las normas del lugar de destino y tienen la posibilidad de crear vínculos con las niñas y los niños autóctonos. Por esta razón, la educación, además de ser un derecho, también es una obligación, puesto que constituye un mecanismo privilegiado de reproducción social y cultural (Suárez-Orozco y Suárez-Orozco 2008:40, 76).

Ciertamente las niñas y los niños migrantes tienen derecho a la educación o la salud, pero también tienen derecho a estar protegidos de la discriminación y a opinar y asociarse. En otras palabras, la propia Convención de los Derechos del Niño (en adelante CDN) establece que existen diferentes áreas de la vida infantil y todas son necesarias y complementarias, ninguna es más importante que otra. En este sentido, los derechos de la CDN se han clasificado en provisión (educación, salud), protección (no discriminación) y participación (opinión, asociación) (Alfageme et al. 2003). Para efectos analíticos, en este artículo consideraremos como derechos sociales (redistribución igualitaria, Fraser 2008) el ámbito de la provisión; mientras que como derechos culturales (reconocimiento e identidad, Fraser 2008), la protección y participación. Integralmente todos facilitan la integración social y cultural.

\section{Contexto de las Migraciones en Chile}

Las cifras sobre población migrante en Chile son aproximativas y han ido en aumento durante los últimos años. Según estimaciones del Departamento de Extranjería y Migración (Rojas y Silva 2016:20), en Chile residen 477.450 personas extranjeras, de este número, $21,65 \%$ corresponde a población infantil y adolescente entre los 0 a 19 años ${ }^{3}$. La población migrante se concentra en las grandes ciudades del país ${ }^{4}$ y provienen principalmente de 
países latinoamericanos (Perú 37,1\%, Argentina $14,3 \%$, Bolivia $8,8 \%$, Colombia $6,0 \%$, Ecuador $5,0 \%$ y España $3 \%$ ), destacándose mayor presencia de mujeres $(52,9 \%)$ que hombres $(47,1 \%)$. Para el 2016, la matrícula de estudiantes migrantes llegó a 60.844 , equivalente al $1,7 \%$ de la matrícula total (Joiko y Vásquez 2016:6). Generalmente, las niñas y los niños migrantes estudian en establecimientos educacionales públicos.

Los derechos de las niñas y los niños migrantes en Chile están garantizados en una serie de instrumentos de carácter administrativo (Circulares u Oficios ministeriales), como una forma de responder a los tratados internacionales de derechos humanos; sin embargo no existe una política pública que esté diseñada particularmente para garantizar derechos de la infancia migrante (UNICEF 2012). Por un lado, la llamada "Ley de Extranjería" promulgada en 1975 (Decreto Ley No 1094) e inspirada en una ideología de la seguridad nacional no considera el tema de la integración de las niñas y los niños migrantes, si bien se estaría elaborando un nuevo Proyecto de Ley de Migraciones inspirado en el enfoque de derechos (Rojas y Silva 2016). Por otro lado, desde la política pública de infancia también se está legislando para actualizar las leyes. Si bien estas nuevas leyes serán un avance, desde un punto de vista crítico, aún resultan insuficientes para ser consideradas como una verdadera política pública que garantice los derechos de las niñas y los niños migrantes en Chile (Pavez-Soto 2014).

\section{Apuntes Metodológicos}

El trabajo de campo de esta investigación se realizó a través de la Oficina de Protección de Derechos de la Infancia (OPD) de Recoleta, donde conocimos algunas organizaciones de migrantes que nos contactaron con familias para realizar las entrevistas (técnica de bola de nieve). La metodología del estudio fue de tipo cualitativo. Se realizaron 12 entrevistas a niñas y niños de 6 a 14 años de edad, algunos nacidos en Chile (pero sus progenitores son extranjeros) y otros en Perú. La información fue categorizada mediante el uso del programa informático Atlas.Ti y analizada con la técnica del "Análisis crítico del discurso" (Valles 1997). También se consideraron los aportes del enfoque metodológico focalizado en la infancia (child-focused), el cual garantiza los derechos de las niñas y los niños que participan en un proceso de investigación social (James y James 2012:10 y ss). Por eso, adecuamos las preguntas de las entrevistas a la edad de cada niña o niño entrevistado, incluimos juegos y descansos y se aplicó un protocolo ético a través de la firma de Consentimiento y Asentimiento Informado.

\section{Integración social: derechos sociales}

Este conjunto de derechos apunta a poseer, recibir o tener acceso a ciertos bienes y/o servicios que garantizan un desarrollo integral de las niñas y los niños en el aspecto físico, intelectual, afectivo y psíquico y de bienestar en condiciones de vida dignas como seres humanos (Alfageme et al. 2003:31). Por extensión, permiten una redistribución igualitaria (Fraser 2008) y la respectiva integración social en la sociedad chilena.

\section{Derechos de provisión}

En Chile, cada vez más comienzan a nacer niñas y niños de madres y padres extranjeros, por ello resulta de vital importancia que puedan ejercer el derecho al nombre y la nacionalidad (Art. $7 \mathrm{CDN}$ ). En nuestro contexto sociojurídico puede ser la "puerta de entrada" para ejercer otros derechos (UNICEF 2012)5. Todas las niñas y los niños entrevistados (tanto quienes nacieron aquí como en sus países de origen) tienen sus documentos de identidad y están en situación administrativa regular.

Un aspecto clave en las condiciones sociales de vida se refiere al derecho a la salud (Art. 24 y $39 \mathrm{CDN}$ ), donde las políticas públicas son cruciales para garantizar u obstaculizar su ejercicio ${ }^{6}$. Según UNICEF (2012:205) las niñas y los niños migrantes habitualmente desconocen cómo poder acceder a los centros de salud locales. Según datos oficiales (MIDESO 2012), las niñas y los niños migrantes de Recoleta están cubiertos por el Fondo Nacional de Salud (FONASA), y solo algunos $(14,9 \%)$ han padecido alguna enfermedad. En nuestro estudio hallamos que la mayoría de niñas y niños conocen el tipo de sistema de previsión de salud que tienen, ya sea público y, en algunos casos, privado.

Generalmente, el nivel socioeconómico familiar determina que las niñas y los niños migrantes puedan o no ejercer su derecho a disponer de un nivel de vida digno y lograr un desarrollo integral (Art. 27 CDN). Según Gaitán (1999), el artículo $\mathrm{N}^{\circ} 27$ de la CDN establece que es responsabilidad 
de las familias el bienestar infantil; lo que se critica como una tendencia hacia la privatización de las responsabilidades públicas que el Estado y la sociedad en su conjunto tienen como garantes de los derechos de la infancia. De acuerdo a cifras oficiales (MIDESO 2012), un porcentaje importante de la población infantil migrante en Chile vive en contextos de pobreza $(15,4 \%)$ y un número no menor en condiciones de pobreza extrema $(4,4 \%)$. En la comuna de Recoleta en particular, las familias migrantes viven en barrios altamente segregados, las condiciones materiales de la vivienda son precarias, algunas sufren situaciones de hacinamiento (por ejemplo, a veces, una habitación de una casona antigua o cité es usada como la vivienda familiar) y un número considerable $(21,3 \%)$ de niñas y niños comparte su dormitorio (MIDESO 2012). En nuestro estudio encontramos las situaciones descritas anteriormente $\mathrm{y}$, además, donde se comparte la misma cama, lo que evidencia la vulneración de este derecho: "duermo con mi hermano en un colchón y mi mamá con su pareja en el otro (Camilo, 12 años, peruano)".

Como ya se dijo, la escuela es uno de los espacios clave en el proceso de integración social que viven las niñas y los niños migrantes, especialmente en el ámbito local, siendo además la educación un derecho (Art. 28 y $29 \mathrm{CDN}$ ). El sistema educativo chileno se destaca por su cobertura, pero, lamentablemente también por la segreación, por eso, las niñas y los niños migrantes se concentran en determinados establecimientos educacionales públicos (Joiko y Vásquez 2016). Las cifras oficiales (MIDESO 2012), muestran que en Recoleta, las niñas y los niños migrantes estudian en colegios municipales (43\%) y subvencionados (57\%), aunque hay casos de quienes aún no pueden acceder (34,9\%). Según el Diagnóstico comunal (OPD Recoleta 2013), conocidas son las Escuelas "Alejandro Flores", "Arturo Pérez Canto" y "Anne Leonor Roosevelt" por acoger a estudiantes migrantes. En nuestro estudio encontramos que las niñas y los niños migrantes, a pesar de algunas dificultades iniciales, acceden a la educación formal; generalmente sus escuelas están ubicadas cerca de sus domicilios, por lo tanto, generan vínculos sociales de amistad en donde espacio escolar y barrio coinciden.

Yo estudio en escuela República de Paraguay. El trato es bueno, los profesores conversan. Tengo compañeros de otros países, bolivianos, peruanos y tengo uno colombiano. Me gusta estar con ellos, son buenos (Jorge, 8 años, chileno).

Respecto a la comparación entre la educación del país de origen y de destino, se denota cierta idealización y nostalgia de la escuela de "allá": la tierra dejada es vista como un paraíso perdido, en contraste con la aparente frialdad de la escuela de “acá" (Moscoso 2008), donde se enfrentan situaciones difíciles, como experiencias de discriminación y el desafío de la adaptación.

Sobre la discriminación que sufren las niñas y los niños migrantes en la escuela, el Diagnóstico comunal (OPD Recoleta 2013:29) constató que un alto porcentaje (49\%) siente distancia de sus compañeras y compañeros de clase y del cuerpo docente, técnico y directivo. Ya se había registrado (Pavez-Soto 2012b; Tijoux 2013) que la nacionalidad opera como un "estigma" social, algo constatado también en este estudio.

Me molestan en el colegio porque soy peruano y me dicen así 'peruano, peruano' siempre me molestan (...) Lo he conversado con mi mamá y me dice que iba a ir al colegio y que le iba a decir a la tía todo lo que pasa. Fue, sí, eso, que lo iban a ver y conversar con los niños, pero sigue igual todo, igual me ha afectado un poco para estudiar (Camilo, 12 años, peruano).

Sin duda, las situaciones de discriminación afectan la acogida y la adaptación de las niñas y los niños migrantes en la escuela, considerando que ésta cumple una función socializadora durante el proceso de integración (Suárez-Orozco y SuárezOrozco 2003):

La escuela donde voy ahora es buena, sí, me gusta, pero la escuela donde iba en Perú era más bacán, porque todos eran peruanos (...) me gusta ir al colegio por los amigos, para compartir (Daniela, 14 años, peruana).

Un último aspecto del ámbito de la protección se refiere a garantizar el derecho al juego, la recreación y el descanso (Art. $31 \mathrm{CDN}$ ). Durante la etapa de la infancia, el juego constituye una forma de aprendizaje y desarrollo social, cultural e identitario. A través de los juegos, las niñas y los niños ponen 
en práctica representaciones sociales y simbólicas sobre su cultura. Por ejemplo, un estudio (Quecha 2014:49) comprobó que las niñas y los niños de familias migrantes transnacionales entre México y Estados Unidos simbolizan la migración a través de sus juegos, especialmente mediante uno llamado "del norte" donde representan el "cruce" de la frontera: algunos niños son policías, otros "mojados" y las niñas, esposas o mamás que lloran. En nuestro estudio hallamos que las niñas y los niños tienen serias dificultades para ejercer su derecho al juego, principalmente por las condiciones de precariedad material en las que viven. Por ejemplo, disponen de pocos lugares para jugar, en general, lo hacen en la calle o en los pasillos de los cités, enfrentándose a situaciones de riesgo (son lugares poco seguros) y conflictos con otros actores por el uso de los espacios comunes. Por lo tanto, un hallazgo de este estudio es que el riesgo que enfrentan las niñas y los niños migrantes a vivir situaciones de violencia y/o discriminación en sus propios barrios es un factor obstaculizador para el ejercicio de sus derechos, no solo al juego, sino también, por extensión a los derechos vinculados a la protección.

Yo no juego acá, porque si yo salgo, ellos [mis hermanos] salen, están los curaos, hablan idioteces, ellos les enseñan, comienzan a repetirlo, no me gusta eso, por eso yo no salgo (Diego, 13 años, peruano).

Desde el punto de vista subjetivo, el análisis de riesgo a vivir situaciones de violencia y/o discriminación -por ejemplo, cuando las niñas y los niños migrantes entrevistados salen a jugar a la calle- puede ayudarnos a interpretar las diversas estrategias que se utilizan para enfrentar ese riesgo. Una forma de enfrentar esas situaciones de riesgo sería el no salir a jugar a la calle y optar por juegos virtuales.

Juego Friv en mi escuela, yo voy a jugar a los juegos de [la calle] Río de Janeiro, también al Parque que está un poco lejos, voy cuando me porto bien. También juego fuera de mi casa (Sebastián, 6 años, chileno).

Se destacan los parques públicos de Recoleta, como el Parque Cerro Blanco, el Parque Bicentenario de la Infancia o aquellos aledaños, como el Parque Forestal, donde las niñas y los niños migrantes puedan ejercer su derecho al juego y la recreación en el ámbito local, porque están ubicados en el barrio, son accesibles y gratuitos. Además, ahí las niñas y los niños tienen la posiblidad de interactuar con otros grupos sociales de la comunidad local, contribuyendo a su integración. "Está el Parque Forestal, es como lo más cercano" (Andrés, 14 años, chileno).

\section{Integración cultural: derechos culturales}

Este ámbito de derechos está relacionado con comprender que las niñas y los niños migrantes deben contar con una protección especial dada su doble condición de vulnerabilidad: por origen nacional y edad, que se acentúa con la condición de género y en situaciones de pobreza o exclusión. Igualmente, los derechos de participación contemplan una visión de las niñas y los niños migrantes como sujetos de derechos (James y James 2012) y de representación (Fraser 2008; Pajares 2005). Como ya se dijo, este ámbito se asocia a la reivindicación de reconocimiento e identidad de sujetos que históricamente han sido considerados como minorías respecto al poder y han estado excluidos por razones culturales. Garantizar estos derechos implica concebir a las niñas y los niños migrantes como sujetos con derechos culturales.

\section{Derechos de protección}

Las niñas y los niños migrantes tienen derecho a estar protegidos de toda forma de discriminación basada en criterios tales como raza, sexo, religión, origen nacional, étnico, posición económica u otra condición (Art. $2 \mathrm{CDN}$ ). Como ya se ha visto, además de la pobreza y la exclusión, las niñas y los niños migrantes sufren de la violencia simbólica que implica la discriminación racial por su origen nacional, color de piel, acento o fenotipo (UNICEF 2012:236; Pavez-Soto 2012b; Tijoux 2013). En una investigación de CEN-OPCION (2012:33-35) el $48,1 \%$ de las niñas y los niños chilenos encuestados dijo que frecuentemente oyen comentarios negativos sobre las niñas y los niños peruanos al interior de su grupo de pares y un 19,2\% no aprueba que las niñas y los niños migrantes tengan acceso a derechos sociales; esta hostilidad es mayor en Santiago que en otras regiones del país. En nuestro estudio encontramos que la mayoría de las niñas y 
los niños migrantes, nacidos aquí o en sus lugares de origen, ha sufrido algún tipo de discriminación en sus colegios y barrios.

Algunos niños del colegio me tratan mal, pero me siento bien estando acá, a pesar de que me molesten algunos, mis compañeros de curso, algunos son molestosos, pero bueno (Diego, 13 años, peruano).

En el Diagnóstico municipal (OPD Recoleta 2013:32) se identificó que las niñas y los niños migrantes tienen una sensación de desprotección y refieren haberse sentido discriminados debido a su nacionalidad, sobre todo al inicio de su llegada a la escuela.

\section{Derechos de participación}

Un aspecto clave de la participación es el derecho a la opinión y que sea tomada en cuenta en los asuntos que les afectan (Art. $12 \mathrm{CDN}$ ). El conocer si las niñas y los niños migrantes participan (opinando y/o decidiendo) sobre el proceso migratorio familiar refleja las relaciones generacionales de poder que se viven al interior de las familias (Pavez-Soto 2011). Cuando no son informados ni consultados sobre el proceso migratorio familiar evidencia una condición específica de desigualdad por edad. En nuestro estudio encontramos situaciones donde las niñas y los niños sí fueron informados, consultados y/o decidieron al respecto y casos donde esto no ocurrió:

Me preguntaron si yo quería venir a Chile. Cuando se vino ella, quería que yo me viniera, pero yo no quise y luego cuando ya estaba preguntando, el año pasado, antes de que se viniera a Perú, me estaba preguntando y ya, sí (Daniela, 14 años, peruana).

Asimismo, las niñas y los niños, sin distinción de edad ni sexo, opinan al interior de sus familias sobre temas domésticos. Algunos niños perciben que el poder opinar reafirma el sentido de pertenencia a su familia y, por extensión, a una comunidad, confirmando que el derecho a la opinión es una reivindicación por el reconocimiento (Fraser 2008) (soy parte de algo y opino sobre ese algo). "Si, me preguntan '¿quieres ir o quieres quedarte'?, me gusta, porque pueden saber lo que me pasa (Leo, 10 años, chileno)".

Otro aspecto clave de la participación, se vincula con el derecho a la libertad de asociación y de celebrar reuniones pacíficas (Art. $15 \mathrm{CDN}$ ). En nuestro estudio, comprobamos que el ejercicio de este derecho está relacionado con variables, tales como el origen nacional -algunas niñas participan en bailes folclóricos-, y los roles de género vigentes, porque solo los varones participan en el Grupo de Futbol "Unión Purísima Loreto" (Centro Cultural Verdaguer, Barrio Bellavista). Para ellas y ellos el participar en una asociación facilita la socialización entre pares y fomenta el sentido de identidad y pertenencia a los territorios locales. "Participo en lo del futbol, desde julio. Ahí hay niños de otros países también, la relación es cercana" (Leo, 10 años, chileno).

\section{Derechos de identidad}

Las niñas y los niños migrantes, en tanto, pertenecientes a minorías étnicas, religiosas, lingüísticas o indígenas tienen derecho a tener su propia vida cultural, religión e idioma (Art. 30 CDN). La integración, a diferencia de la asimilación, implica que ejerzan el derecho a conocer su cultura de origen y que esto suceda en el contexto local, de este modo, se refuerza una identidad y un sentido de pertenencia transnacional. Las niñas y los niños entrevistados identifican una serie de prácticas culturales y costumbres que provienen de sus lugares de origen y que son realizadas y reconstruidas en Recoleta, como la celebración de las Fiestas Patrias, fiestas de fin de año a la manera de "allá", canciones y bailes típicos y especialmente las formas de preparación de la comida (Imilan 2014). "Seguimos cocinando como en Perú" (Jorge, 8 años, chileno).

Tal como señalábamos anteriormente, desde la antropología se entiende que la infancia es un fenómeno construido sociohistórica y culturalmente (Moscoso 2008), también veíamos que la integración de las niñas y los niños migrantes no solo implica el ejercicio de sus derechos, sino, también, la integración en las formas culturales de "ser niña o niño, aquí y ahora" (Alegre 2007:9; Feixa 2006). Respecto a los estereotipos de género, emerge un discurso de reconocimiento hacia las formas de ser niña en Chile vinculadas a la rebeldía. Por otro lado, se hace una crítica moral de un problema social (el embarazo 
precoz); recordemos que según los roles de género vigentes, las niñas y las mujeres son las portadoras de la moral de sus familias y comunidades (Lamas 1999), y en contextos migratorios, esta función se acentúa (Levitt 2009).

[Los chilenos son] bacanes, pero más las mujeres (Daniela, 14 años, peruana). Las chilenas son más rebeladas y tienen hijos a los quince (Diego, 13 años, peruano).

Por su parte, los niños chilenos son vistos con similitudes respecto a los niños extranjeros, se destaca el futbol -elemento clásico de la masculinidad contemporánea- como un espacio de encuentro. A los niños chilenos también se les atribuye un comportamiento rebelde en su relación con las personas adultas. Estos discursos dejan entrever que las niñas y los niños migrantes asocian la rebeldía como uno de los rasgos de "ser niña o niño, aquí y ahora (en Chile)". Entonces, un proceso de adaptación a las formas culturales locales, sería imitando dicha rebeldía, desintegrándose de los modos de ser persona adulta, ya sea chilena o migrante.

Nos llevamos bien porque jugamos a la pelota (Leo, 10 años, chileno).

Los chilenos son más rebelados en cierta edad. Su papá está un rato, lo aconseja y los chilenos no toman esa palabra. Pero los peruanos sí, porque saben las consecuencias (Diego, 13 años, peruano).

Con todo, las niñas y los niños migrantes expresan que comparten valores de su cultura de origen y de la cultura chilena, de esta última, especialmente el lenguaje, en tanto medio de comunicación y mecanismo privilegiado de reproducción cultural. Entendiendo que las culturas son producciones desiguales e incompletas (Bhabha 2002), de ahí que sus identidades sean locales, híbridas y globales (Levitt 2009).

Es posible sostener que el ejercicio de los derechos tiene consecuencias directas en los procesos de integración de las niñas y los niños migrantes en nuestro país. Resulta interesante comprobar cómo la vivencia cotidiana de los vínculos, los silencios, el fracaso escolar, la violencia o la distancia con sus pares en la escuela y en el barrio es de vital importancia para la integración social y cultural, reconociendo la condición de vulnerabilidad en que las niñas y los niños migrantes son situados en nuestro contexto.

\section{Conclusiones}

El contexto local es clave en el proceso de integración sociocultural de las niñas y los niños migrantes, puesto que ahí ejercen sus derechos cotidianamente: en sus familias, grupos de pares, escuela, barrios y territorios. Por eso, la institucionalidad política y la comunidad local constituyen los principales garantes. Como primera conclusión y hallazgo del estudio, se reconoce que en el caso de Recoleta hay voluntad política para la integración de las niñas y los niños migrantes. Sin embargo, aún se observan diversas situaciones donde sus derechos no son ejercidos, dificultando su integración.

Como segundo hallazgo de nuestro estudio, podemos decir que los derechos relativos al ámbito de la provisión, tales como a la salud y educación se ejercen en mayor medida que a gozar de un nivel de vida óptimo, a integrarse plenamente en el ámbito escolar y al juego. El nivel de vida en que viven las niñas y los niños migrantes generalmente es de pobreza y exclusión, en un escenario de escasas políticas públicas que ayuden a revertirlo. Si bien se cumple el acceso a la escolarización, se evidencia la falta de políticas de convivencia escolar ante las situaciones de discriminación, esto último, simultáneamente, vulnera los derechos de protección. Aunque el derecho al juego se ve dificultado en el hogar -por razones de precariedad material-, los parques públicos de Recoleta lo facilitan, pero aún son escasos. Por lo tanto, se concluye que existe integración social vinculada a la redistribución igualitaria, pero aún es muy incipiente y requiere todavía mayores esfuerzos políticos del ámbito local para lograrse.

En tercer lugar, se concluye como otro hallazgo de esta investigación que los derechos relativos a la participación y la identidad se ejercen en el ámbito local de Recoleta, aunque la opinión no siempre es considerada en los asuntos que afectan a las niñas y los niños -por ejemplo, en el proceso de su propia migración-, se destaca la participación en grupos sociales locales que fortalecen el sentido de pertenencia. Las niñas y los niños entrevistados reflexionan críticamente con las formas de "ser niña o niño, aquí y ahora" en Chile. Se deriva que la 
integración cultural relacionada con el reconocimiento e identidad devela conflictos y negociaciones en la construcción de una identidad transnacional.

En este estudio quisimos analizar los alcances y las limitaciones del concepto de integración sociocultural en el contexto local, entendiendo que toda conceptualización teórico-metodológica conlleva implicancias ideológico-políticas, que también es preciso explicitar y analizar. Como ya se dijo, en el contexto estudiado (Recoleta) se identifican esfuerzos incipientes para lograr la integración de las niñas y los niños migrantes, una serie de indicadores institucionales y políticos existentes así lo demuestran, pero aún existen carencias y desafíos. Esta investigación quiere ser un aporte al debate público que existe actualmente en la sociedad chilena a fin de comprender a las niñas y los niños migrantes como sujetos de derecho.

Agradecimientos: Agradezco al Programa FONDECYT de CONICYT el financiamiento entregado para ejecutar el Proyecto de Investigación FONDECYT de Iniciación No 11121295 “La infancia como sujeto de las políticas públicas e intervenciones sociales. El caso de la niñez migrante en el Chile del siglo XXI", dirigido por Iskra Pavez-Soto y patrocinado por la Universidad Alberto Hurtado y la Universidad UCINF. También quiero agradecer a la OPD Recoleta por su apoyo para realizar el estudio, los comentarios de Sònia Parella y las correcciones de los evaluadores anónimos que me ayudaron a mejorar el manuscrito.

\section{Referencias Citadas}

Alegre, M. 2007. Geografies Adolescents a Secundària. Posicionaments Culturals i Relacionals dels i les Joves d'Origen Immigrant. Observatori Català de la Joventut, Barcelona.

Alfageme, E., R. Cantos y M. Martínez 2003. De la Participación al Protagonismo Infantil. Propuestas para la acción. Plataforma de Organizaciones de Infancia, Madrid.

Bhabha, H.K. 2002. El Lugar de la Cultura. Ediciones Manantial, Buenos Aires.

Bhabha, H.K. 2013. Nuevas Minorías, Nuevos Derechos. Notas sobre Cosmopolititsmos Vernáculos. Siglo XXI Editores, Buenos Aires.

CEN-OPCIÓN 2012. Niños, Niñas y Derechos Humanos: Nuevos Actores y Nuevas Visiones. Principales Resultados de la Encuesta Nacional de Derechos Humanos y Niños, Niñas y Adolescentes. LOM, Santiago.

Fraser, N. 2008. La justicia social en la era de la política de identidad: redistribución, reconocimiento y participación. Revista de Trabajo 6:83-99.

Feixa, C. (dir.) 2006. Jóvenes “Latinos” en Barcelona. Espacio Público y Cultura Urbana. Anthropos, Barcelona.

Gaitán, L. 1999. El Espacio Social de la Infancia. Los Niños en el Estado de Bienestar. Comunidad de Madrid, Madrid.

Gaitán, L. 2006. Sociología de la Infancia. Síntesis, Madrid.

García Castaño, F.J., R.A. Pulido Moyano y Á. Montes del Castillo 1993. La educación multicultural y el concepto de cultura. Una visión desde la antropología social y cultural. Revista de Educación 302:83-110.

Gordon, M. 1964. Assimilation in American Life. The Role of Race, Religion and National Origin. Oxford University Press, New York.

Imilan, W. 2014. Restaurantes peruanos en Santiago de Chile: construcción de un paisaje de la migración. Estudios Sociales 48:15-28.
James, A. y A. James 2012. Key Concepts in Childhood Studies. Sage, London.

Joiko, S. y A. Vásquez 2016. Acceso y elección escolar de familias migrantes en Chile: "No tuve problemas porque la escuela es abierta, porque acepta muchas nacionalidades". Calidad en la Educación 45:132-173.

Lamas, M. 1999. Usos, dificultades y posibilidades de la categoría género. Papeles de Población 21:147-178.

Levitt, P. 2009. Roots and routes: Understanding the lives of the second generation transnationally. Journal of Ethnic and Migration Studies 35:225-1242.

Malgesini, G. y C. Giménez 2000. Guía de Conceptos sobre Migraciones, Racismo e Interculturalidad. Ediciones Catarata, Madrid.

Margarit, D. y K. Bijit 2014. Barrios y población inmigrante: el caso de la comuna de Santiago. INVI 8:19-77.

Márquez, F. 2013. De territorios, fronteras e inmigrantes. Representaciones translocales en la chimba, Santiago de Chile. Chungara Revista de Antropología Chilena 45:321-332.

Moscoso, M. 2008. La mirada ausente: Antropología e infancia. http://www.uasb.edu.ec/UserFiles/369/File/PDF/ CentrodeReferencia/Temasdeanalisis2/ninezadolescenciayjuventud/ articulos/Moscoso.pdf (12 de enero de 2016).

MIDESO 2012. Resultados Encuesta CASEN 2011. Bases de datos.

OPD Recoleta 2013. Diagnóstico local de niñez y juventud. Resumen ejecutivo. Manuscrito en posesión de los autores.

Pajares, M. 2005. La Integración Ciudadana. Una Perspectiva para la Inmigración. Icaria, Barcelona.

Pavez-Soto, I. 2011. ¿Quién decide la migración infantil? Niñez y poder en familias peruanas transnacionales. RAYUELA, Revista Iberoamericana sobre Niñez y Juventud en Lucha por sus Derechos 5:103-113. 
Pavez-Soto, I. 2012a. Sociología de la infancia. Las niñas y los niños como actores sociales. Revista de Sociología 27:81-102.

Pavez-Soto, I. 2012b. Inmigración y racismo: experiencias de la niñez peruana en Santiago de Chile. Si Somos Americanos: Revista de Estudios Transfronterizos 12:75-99.

Pavez-Soto, I. 2014. De la reunificación familiar a la migración infantil: los derechos de las niñas y los niños peruanos en Chile. En Regiones Fronterizas, Migración y los Desafíos para los Estados Nacionales Latinoamericanos, compilado por M. Tapia y A. González, pp. 49-274. Editorial RIL, Santiago.

Portes, A., P. Fernández-Kelly y W. Haller 2006. La asimilación segmentada sobre el terreno: la nueva segunda generación al inicio de la vida adulta. Migraciones 19:7-58.

Quecha, C. 2014. Jugar al norte: una representación lúdica de la migración internacional en niños afrodescendientes no migrantes. Alteridades 47:43-52.

Qvortrup, J. 1994. Childhood Matters: An Introduction. En Childhood Matters: Social Theory, Practice and Politics, editado por J. Qvortrup, M. Bardy, G. Sgritta y H. Winstersberger, pp. 14:1-23. Avebury-European Centre Vienna, Viena.

Rojas, N. y C. Silva 2016. La migración en Chile: breve reporte y caracterización. Informe Observatorio Iberoamericano sobre Movilidad Humana, Migraciones y Desarrollo, OBIMID, Madrid.

Silva, R., C. Villalobos y M. Saracostti 2011. Evaluando el nivel de implementación de las políticas de infancia y adolescencia en las Regiones Metropolitana y de Valparaíso: una mirada a la calidad desde la complejidad municipal. Revista Iberoamericana de Estudios Municipales 4:133-161.

Solé, C., T. Sordé, O. Serradell, R. Alcalde, A. Flecha, A. Pettroff, L. Cavalcanti, S. Parella, I. Pavez, E. Santamaría y L. Garzón 2011. Cohesión social: aportaciones científicas y discursos políticos. Revista Internacional de Sociología 69:9-32.

Stefoni, C. 2015. Reconfiguraciones identitarias a partir de habitar el espacio público. El caso de los migrantes esquineros en la ciudad de Santiago, Chile. Chungara Revista de Antropología Chilena 47:669-678.

Suárez-Orozco, C. y M. Suárez-Orozco 2003. La Infancia de la Inmigración. Morata, Madrid.

Tijoux, M. 2013. Niños(as) marcados por la inmigración peruana: estigma, sufrimientos, resistencias. Convergencia 61:83-104.

UNICEF 2012. Los Derechos de los Niños, Niñas y Adolescentes Migrantes, Refugiados y Víctimas de Trata Internacional en Chile. Avances y Desafíos. UNICEF, Santiago.

Valles, M. 1997. Técnicas Cualitativas de Investigación Social. Reflexión Metodológica y Práctica Profesional. Síntesis, Madrid.

Wasserman, T. 2001. ¿Quién sujeta al sujeto? Una reflexión sobre la expresión "el niño como sujeto de derecho". Revista Ensayos y Experiencias 41:60-69.

\section{Notas}

1 El Municipio de Recoleta ocupa el lugar $7^{\circ}$ (de 45), está en fase de consolidación e innovación de sus políticas de infancia, "cuenta con condiciones adecuadas de dotación de personal, recursos financieros, infraestructura, complejidad del entorno, etc., además, posee un nivel avanzado de implementación de políticas de infancia. Debe consolidar sus resultados en los indicadores evaluados, y desarrollar innovaciones en políticas de infancia que permitan maximizar la implementación de una perspectiva integral de derechos" (Silva et al. 2011:141).

2 Durante la época del Imperio Romano se establecía que la infantia duraba desde el nacimiento hasta los 7 años; luego desde los 7 hasta los 12 en el caso de las niñas y 14, para niños, correspondía la pueritia. Posteriormente venía la adulescentia de 12 ó 14 años, hasta los 21 años de edad; a partir de ese momento se entra en la etapa de la plenitud denominada iuventus, la cual dura hasta los 50 años, desde los 50 años y hasta los 72 se denomina gravitas y a partir de los 72 años hasta la muerte, senectus (Wasserman 2001).

3 En las estadísticas oficiales del Departamento de Extranjería y Migración (DEM) sobre población migrante en Chile los tramos de edad que se consideran son: de 0 a 19 años; 20 a $35 ; 36$ a 50; 51 a 65; 66 y más (Rojas y Silva 2016:20). Cabe decir que en esos tramos se excede el tramo de población infantil y se incluye a la adolescente y juvenil (0 a 19 años de edad).

4 La población migrante reside mayormente en las regiones Metropolitana (64,1\%), Antofagasta (7,8\%), Tarapacá $(6,4 \%)$ y Valparaíso (5,5\%) (Rojas y Silva 2016).

$5 \quad$ Al 2011 habían 25.312 niñas y niños nacidos en Chile de madre o padre extranjero (MIDESO 2012). La Constitución política de Chile (Art. 10) señala que son chilenos quienes nacen en territorio chileno (ius solis) (...) con excepción de los hijos de extranjeros transeúntes. Durante años, la categoría «extranjero transeúnte» se equiparó a situación administrativa irregular ocasionando que algunas niñas y niños quedaran inscritos en el Registro Civil como ET (hijo de extranjero transeúnte), sin nacionalidad chilena. En 2014 se instruyó que la situación administrativa irregular de madres y padres no afecta el derecho a nacionalidad de sus hijas e hijos nacidos en Chile (Resolución Exenta No 3207/ 8 de agosto 2014).

6 En 2008 el Ministerio de Salud elaboró una Circular (A14, $\mathrm{N}^{\circ} 3229$ ) que garantiza el derecho a la salud de niñas y niños migrantes y entrega un permiso de residencia temporal. En 2014, se suscribe un Convenio del Ministerio del Interior (Decreto $\mathrm{N}^{\circ} 2410$ ) que permite acceder a las prestaciones del Fondo Nacional de Salud (FONASA) a las personas migrantes desde que inician el proceso de regularización administrativa. 